\title{
¿Por qué sigue siendo tan alta la frecuencia de errores en el cuidado de los pacientes?
} Why are patient care errors still so frequent?

Sin duda, desde hace miles de años ya era conocido que los errores que cometían las personas, formaban parte y eran propios de la naturaleza humana. Por lo tanto, indefectiblemente ocurren con frecuencia, y seguirán ocurriendo en todas las actividades de nuestras vidas. En forma cotidiana, encaramos múltiples acciones, la mayoría son comunes y rutinarias, tomamos decisiones y emitimos nuestro juicio clínico; pero en forma simultánea y por diversos y complejos motivos, cometemos numerosos errores en aspectos o acciones que suponemos conocer. El que comete un error cree que su acción, su juicio o su hipótesis suelen ser adecuados y por lo tanto, esa creencia continuará hasta que nos percatemos que el error indefectiblemente volverá a ocurrir. Ese es el momento oportuno para poder cambiar esa creencia equivocada y reconocer que después de cometer un error lo importante es aprender por qué ocurrió el error y de ese modo, es probable que no se repita. Lamentablemente, esta actitud no es la que predomina en muchas personas ya que suponen que no pasará de nuevo, siendo que sin ninguna duda, el error volverá a ocurrir. Si no adquirimos una actitud crítica en el cuidado de los pacientes, los errores continuarán aumentando y así habrá más eventos adversos que pueden ser de gravedad, incluso en provocar la muerte.

Este tema ha merecido múltiples estudios en distintas disciplinas, pero aún así persisten varios interrogantes acerca de por qué cometemos errores con tanta frecuencia. Las investigaciones se orientaron principalmente hacia dos ejes, el factor humano y la psicología cognitiva.

Los factores humanos han sido estudiados especialmente por ingenieros en áreas tales como la aviación y el control de plantas nucleares. Los psicólogos cognitivos se han concentrado en desarrollar el modelo de la cognición en el ser humano. A través de los avances en las neurociencias se ha logrado conocer mejor los diversos aspectos fisiológicos de la cognición. Asimismo, se pudo determinar que varios errores ocurren por una disfunción o alteración de la función cognitiva. Esto ha llevado a disponer de teorías razonablemente coherentes sobre por qué los humanos frecuentemente cometemos errores y a partir de eso, debemos diseñar las posibles estrategias para disminuir o minimizar su ocurrencia.

En medicina, la alta frecuencia de errores ocurre por diferentes y complejos aspectos. Una de nuestras acciones que incrementa la ocurrencia de errores es que tanto los médicos como otros profesionales del cuidado de la salud, tienen grandes dificultades en enfrentar y aceptar el error humano. En la mayoría de las escuelas de medicina y en las residencias, al igual que en las escuelas de enfermería y farmacia, el tema del error no se trata y de esa forma no llegan preparados cuando ya deben cuidar a los pacientes. Varios artículos, han observado que la tasa de errores médicos son más frecuentes en la residencia.

Para que se pueda lograr una disminución de errores en la práctica médica, el imprescindible primer paso es que se genere un cambio en la cultura tradicional en el abordaje del error en medicina. Esa cultura, que lamentablemente aún sigue siendo muy frecuente en las instituciones de la salud, se basa en el ocultamiento del error y en el castigo o punición a quien lo comete. Este denigrante y no honesto modelo, estaría presente en la medicina desde hace mucho tiempo, y en caso que siga predominando, sin ninguna duda, no será posible reducir los errores. Las evidencias de hoy en día, muestran que si no hay un mayor cambio de cultura, los errores seguirán aumentando.

Asimismo, aun en los países más desarrollados persiste la cultura tradicional y por lo tanto no logran disminuir los errores y sus eventos adversos, siendo que no alcanzan una menor frecuencia, tal como ellos desean. En EE. UU., los errores que ocurren en los cuidados de los pacientes hospitalizados constituyen la tercera causa de muertes anualmente, luego de los trastornos cardíacos y el cáncer. En años recientes, se observó que alrededor de 300000 pacientes fallecen en los hospitales anualmente por causa de errores que llevaron a que el paciente tuviera un evento adverso grave. No hay ninguna otra causa en todo el mundo que alcance esa enorme cantidad de muertos.

El Dr. Lucian Leape de la Universidad de Harvard fue quien por primera vez en la medicina 
conformó un excelente grupo que estudiaron cuáles eran las causas de la mortalidad en pacientes hospitalizados en la Ciudad de New York, publicado en 1991. Luego de unos años en una entrevista mencionó esta pregunta: ¿Cuán peligrosos son los cuidados médicos?, y los comparó con las múltiples situaciones que el ser humano puede pasar en su vida (terremotos, inundaciones, accidentes, etc.); y manifestó que "el riesgo de muerte más peligroso para cualquier persona es internarse en un hospital".

La Organización Mundial de la Salud (OMS) creó en octubre de 2004 una iniciativa especial denominada Alianza Mundial para la Seguridad del Paciente con el deseo de establecer las políticas necesarias, entre otras el cambio de cultura, para mejorar la seguridad de los pacientes. Posteriormente, en 2007 la OMS envío un texto donde se solicitaba que hubiera más investigaciones para mejorar la seguridad del paciente. Asimismo, señaló que más de 10 millones de enfermos que mueren o sufren trastornos graves cada año como consecuencia de "prácticas médicas inseguras".

La iniciativa de la OMS en su Alianza Mundial para la Seguridad del Paciente trajo varios avances en los progresos de acciones y programas especiales para disminuir los errores en el cuidado de los pacientes, debido a los inadecuados sistemas en las instituciones médicas.

El segundo tema que encaró la OMS dentro de la Alianza fue el de la seguridad en los pacientes que se someten a una intervención quirúrgica. Bajo el lema: "Cirugía segura salva vidas" se implementó un programa mundial para disminuir los errores en cirugía y en 2008 se difundieron las guías para poder alcanzar esa meta (WHO guidelines for safe surgery). Los errores en cirugía son frecuentes, aun cuando se ignora su verdadera prevalencia, y afectan a millones de personas. Se calcula que no menos de 250 millones de cirugías mayores se realizan anualmente en el mundo, por lo cual las posibilidades que ocurran descuidos que ocasionen errores son muy altas.

Por lo tanto, es absolutamente necesario que los profesionales de la salud continúen en el camino que puede llegar el cambio de una nueva cultura, donde los errores se aceptan y de esa forma se aprende como ocurrieron con el fin de disminuirlos. Esa cultura llevará a generar mejores sistemas en los hospitales mediante las medidas de prevención de errores para que el error no llegue a las personas.
Lamentablemente, la gran mayoría de los hospitales de nuestro país no se ocupan del problema de los errores y, no cuentan con un Comité de Seguridad del Paciente. Por lo cual no logran mejorar los sistemas que son múltiples; y así persisten las notorias deficiencias que incrementan que los errores ocurran con mucha frecuencia.

Es muy probable que esto se debe a que muchas instituciones médicas no avanzaron en la seguridad del paciente ya que no reconocen o no aplican la nueva cultura en el enfoque del error. Además, no suelen tener en cuenta que los errores ocurren principalmente a causa de los sistemas hospitalarios deficientes, y no en el individuo.

Debemos continuar bregando para mejorar con todo esfuerzo los cuidados de los pacientes hospitalizados, solo así vamos a poder respetar esa milenaria acción que dice "primero no dañar".

José María Ceriani Cernadas Editor

http:/ / dx.doi.org/10.5546/aap.2019.282

Texto completo en inglés: http:/ / dx.doi.org/10.5546/ aap.2019.eng.282

Cómo citar: Ceriani Cernadas JM. ¿Por qué sigue siendo tan alta la frecuencia de errores en el cuidado de los pacientes?. Arch Argent Pediatr 2019;117(5):282-283.

\section{REFERENCIAS}

- DiNardo D, Tilstra S, McNeil M, Follansbee W, et al. Identification of facilitators and barriers to residents' use of a clinical reasoning too. Diagnosis (Berl). 2018;5(1):21-8.

- Sexton JB, Adair KC, Leonard MW, Frankel TC, et al. Providing feedback following Leadership WalkRounds is associated with better patient safety culture, higher employee engagement and -lower burnout. BMJ Qual Saf. 2018;27(4):261-70.

- Bradley EH, Brewster AL, McNatt Z, Linnander EL et al. How guiding coalitions promote positive culture change in hospitals: a longitudinal mixed methods interventional study. BMJ Qual Saf. 2018;27(3):218-25.

- Sammer C, Hauck LD, Jones C, Zaiback-Aldinger J, et al. Examining the relationship of an all-cause harm patient safety measure and critical performance measures at the frontline of care. J Patient Saf. 2018. Epub ahead of print Feb 7, 2018.

- Austin JM, Pronovost PJ. "Never events" and the quest to reduce preventable harm. Jt Comm J Qual Patient Saf. 2015;41(6):279-88.

- Cheung R, Roland D, Lachman P. Reclaiming the systems approach to paediatric safety. Arch Dis Child. 2019;pii: archdischild-2018-316401. Epub ahead of print Feb 23, 2019.

- Stockwell DC, Landrigan CP, Toomey SL, Loren SS, et al. Adverse events in hospitalized pediatric patients. Pediatrics. 2018;142(2):e20173360. 